\title{
Laser Shock Processing influence on local properties and overall tensile behavior of friction stir welded joints
}

\author{
Mihaela Iordachescu ${ }^{\mathrm{a}, *}$, Andrés Valiente ${ }^{\mathrm{a}}$, Luis Caballero ${ }^{\mathrm{a}}$, Danut Iordachescu ${ }^{\mathrm{b}}$, \\ José Luis Ocaña ${ }^{b}$, Juan Antonio Porro ${ }^{\text {b }}$
}

\begin{abstract}
A B S T R A C T
Based on laser beam intensities above $10^{9} \mathrm{~W} / \mathrm{cm}^{2}$ with pulse energy of several Joules and duration of nanoseconds, Laser Shock Processing (LSP) is capable of inducing a surface compressive residual stress field. The paper presents experimental results showing the ability of LSP to improve the mechanical strength and cracking resistance of AA2024-T351 friction stir welded (FSW) joints. After introducing the FSW and LSP procedures, the results of microstructural analysis and micro-hardness are discussed. Video Image Correlation was used to measure the displacement and strain fields produced during tensile testing of flat specimens; the local and overall tensile behavior of native FSW joints vs. LSP treated were analyzed. Further, results of slow strain rate tensile testing of the FSW joints, native and LSP treated, performed in $3.5 \% \mathrm{NaCl}$ solution are presented. The ability of LSP to improve the structural behavior of the FSW ioints is underscored.
\end{abstract}

\section{Introduction}

Starting early 1920 s, aerospatial structures have used aluminium alloys due to their high strength-to-weight ratio developed through precipitation hardening. However, the presence of precipitates within these alloys leads to poor corrosion resistance [1-3]. Therefore the structural use of aluminium alloys requires the development of treatments to improve their corrosion resistance.

It was proved that traditional fusion welding techniques are not able to provide qualitative joints made of high strength aluminium alloys because of the high power concentration needed; moreover, these alloys exhibit the tendency for both solidification and liquation cracking $[2,4]$.

Since in the early ' 90 s the new technique of Friction Stir Welding (FSW) emerged, it was clear that it is very suitable for joining of aluminium alloys [1-6]. FSW brings the metal into a plastic state at a temperature below its melting point, and forms the welded joint between the two parts, stirred together under pressure [7].

Nevertheless, FSW has its limitations. It was almost generally reported that the FSW aluminium joints exhibit a slight undermatch in strength with respect to the base metal [8-9]. Moreover, the residual stress field developed during the welding process is a critical feature for the service performance of the welded joint, since it can favour stress corrosion cracking (SCC) in the presence of specific environments $[3,8]$.

Previous investigations have shown that the maximum residual stresses are less than those induced by the traditional welding processes [10]. Across the weld region the residual stress distribution reveals an " $\mathrm{M}$ "-like shape, asymmetric with respect to the weld centerline, with the largest longitudinal stress components oriented along the weld line, taking values ranging from $15 \%$ to $30 \%$ of the parent material yield stress [10-12].

In the last decade it has been reported $[4,8,13-15]$ that peening techniques like Laser Shock Processing (LSP) are local surface treatments capable of inducing deep-enough compressive residual stress to moderate or even to neutralize the welding residual tensile stress field.

LSP consists of the application of a high intensity pulsed Laser beam (irradiance, $\mathrm{I}>10^{9} \mathrm{~W} / \mathrm{cm}^{2}$, and pulse duration, $T<50 \mathrm{~ns}$ ) on a metallic target forcing a sudden surface vaporization that immediately produces a high temperature and density plasma capable of inducing a mechanical shock wave which propagates into the material [10-11].

The main objective of the investigation was to assess the ability of LSP in modifying the overall and local mechanical behavior of the AA 2024-T351FSW joints. In this view, a video-image based system was used for displacement and strain field measurement during the tensile testing of the FSW joints, native and LSP treated. The plastic strain concentration revealed by this full-field experimental data is 
explaining the joint failure in the retreating side (RS) of the thermomechanically affected zone (TMAZ).

Another subsequent outcome of the investigation was to determine the local tensile behavior of the joint different zones, native or superficially treated by LSP. Finally, the LSP effect on stress corrosion cracking (SCC) of the FSW joints is also discussed on the basis of slow strain rate tensile tests carried out in an aggressive medium.

\section{Experimental approach}

The AA2024-T351 aluminium alloy is an Al-Cu-Mg alloy which has been solution heat-treated, control stretched and naturally aged. It was selected due to high strength and suitable fracture toughness; its chemical composition is given in Table 1[16]. The investigation was made on $4 \mathrm{~mm}$ thick plates, in as received T351 conditions, without any additional surface treatment to increase the corrosion resistance.

The FSW samples were made at DEM-Instituto Superior Tecnico, TULISBON, Lisbon, using specialized equipment. The welding direction was perpendicular to the rolling direction of the AA2024 plates; the dimensions of the samples after welding were $200 \mathrm{~mm} \times 250 \mathrm{~mm}$. The main processing parameters for performing the FSW butt welds are presented in Table 2 .

No cracks or indications of other surface-open flaws have been revealed after the visual inspection of the samples.

Tensile specimens (conventional dog bone coupons of loading direction normal to the weld), with a gauge length of $50 \mathrm{~mm}$ and a gauge width of $12.5 \mathrm{~mm}$ were machined, in accordance with ASTM $\mathrm{E} 8$, from the FSW joint panels and native base metal plates. The Laser Shock Processing was applied on both faces of the specimens, on a total length of $57 \mathrm{~mm}$ (Fig. 1). Untreated FSW specimens and base metal specimens were also tensile tested for comparison.

According to ISO 7539-7 prescriptions, the same type of specimens was used for the slow strain rate tensile tests, carried out in an aggressive environment, to measure the joints susceptibility to stress corrosion cracking.

The Laser Shock Processing was performed with a Nd:YAG laser emitting at $1064 \mathrm{~nm}$ (fundamental) and $532 \mathrm{~nm}$ (1st harmonic) wave lengths, at UPM Laser Centre, Universidad Politécnica de Madrid. A Q-switch device was used to produce the nanoseconds regime. The treatment area was covered with an approximately $1 \mathrm{~mm}$ thick laminar layer of flowing water, and the laser beam was brought into it by means of a set of mirrors arrangement. No other absorbing coating was used [14]. A 2D motion system was used to control the specimen position against the laser beam and generate the pulse swept (Fig. 1b). The LSP surface sweeping strategy, namely the equivalent number of laser pulses per unit area of processed surface was obtained by controlling the system velocity and the pitch overlapping. The processing parameters are given in Table 3.

Tensile testing was performed at Materials Science Dpt., E.T.S.I. Caminos, Universidad Politécnica de Madrid, at room temperature on a $200 \mathrm{kN}$ servo-hydraulic universal testing machine using a constant crosshead speed of $1 \mathrm{~mm} / \mathrm{min}$. A Video Image Correlation system (VIC-2D) was used additionally to conventional clip-on extensometers to assess the strain and displacement fields during tensile loading of the LSP treated and untreated FSW specimens.

VIC-2D is a displacement and strain measurement technique capable to analyze the digital images data taken during a tension test of a

Table 1

Nominal weight percentage for AA2024 chemical composition.

\begin{tabular}{lllllllll}
\hline $\mathrm{Al}$ & $\mathrm{Cr}$ & $\mathrm{Cu}$ & $\mathrm{Fe}$ & $\mathrm{Mg}$ & $\mathrm{Mn}$ & $\mathrm{Si}$ & $\mathrm{Ti}$ & $\mathrm{Zn}$ \\
\hline $\mathrm{Bal}$ & 0.10 & $3.4-4.9$ & 0.50 & $1.2-1.8$ & $0.3-0.9$ & 0.50 & 0.15 & 0.25 \\
\hline
\end{tabular}

Table 2

FSW processing parameters.

\begin{tabular}{ll}
\hline FSW parameters & \\
\hline Pin length, mm & 4.17 \\
Tilt angle, & 0 \\
FSW control & Vertical force control \\
Vertical downward forging force, kgf & 890 \\
Rotation speed, rpm & 1000 \\
Rotation direction & $\mathrm{CW}$ \\
Travel speed, mm/min & 300 \\
Plunge speed, $\mathrm{mm} / \mathrm{s}$ & 0.1 \\
Dwell time, $\mathrm{s}$ & 8 \\
Rolling direction vs. weld line & Perpendicular \\
\hline
\end{tabular}

flat specimen. The camera is placed perpendicular to the specimen surface and its calibration requires only the determination of the scale factor. Consecutive digital images are used to monitor the changes of a speckle pattern, previously applied on the specimen surface. The system uses an iterative spatial domain cross-correlation algorithm to track the speckle pattern movement during loading. The error in the in-plane strain and displacement measurements due to out-of-plane motion of the target surface is proportional to the ratio of the out-of-plane displacement to the focal length of the camera, and is typically less than $1 \%$.

The system was used to assess the longitudinal strain distribution over an area of interest (AOI) defined on the specimen surface, containing the FSW joint, LSP treated or not, during tensile loading. The results were used to obtain strain averages data vs. testing time, using "virtual extensometers", on gauge lengths comparable with the lengths of different weld regions. The average strains were mapped to the corresponding global stress levels applied by the testing machine, assuming that the transversely loaded FSW specimens were in iso-stress configuration.

\section{Results and discussion}

\subsection{Welded joint microstructure}

Standard procedures were used to prepare the metallographic specimens. Different etching reagents were used to obtain high

a

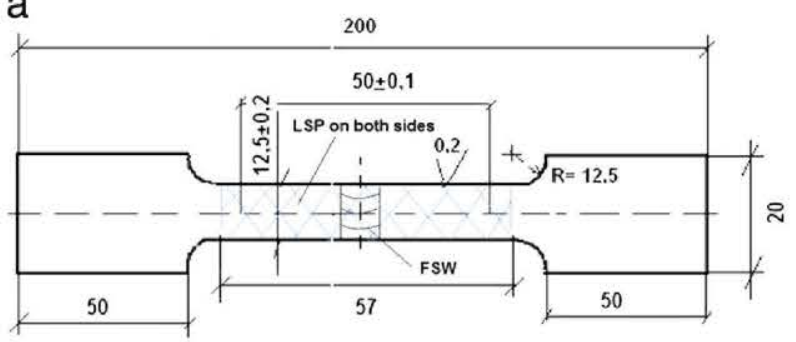

$y$ - longitudinal direction of FSW

$x$ - transverse to FSW (rolling plate direction)

b

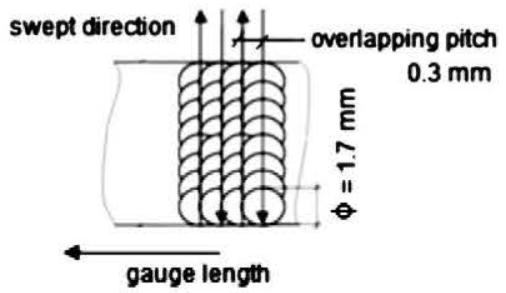

Fig. 1. Sketch of the FSW and FSW with LSP specimen used for tension and SSC testing: a) specimen geometry with dimensions in mm; b) LSP surface sweeping strategy. 
Table 3

LSP processing parameters.

\begin{tabular}{ll}
\hline LSP parameters & \\
\hline Wavelength, $10^{-9} \mathrm{~m}$ & 1064 \\
Frequency, $\mathrm{Hz}$ & 10 \\
Energy, $\mathrm{J} / \mathrm{pulse}$ & 2.8 \\
Pulse duration, $10^{-9} \mathrm{~s}$ & $\sim 9$ \\
Spot diameter, $\mathrm{mm}$ & $1.679-1.847$ \\
Overlapping, pulses $/ \mathrm{cm}^{2}$ & 1600 \\
Confining medium & Water jet \\
\hline
\end{tabular}

contrast images of each FSW, LSP treated microstructure: first, Keller (Fig. 2a and f-h); second, $10 \mathrm{~g} \mathrm{NaOH}, 5 \mathrm{~g} \mathrm{~K}_{4}\left[\mathrm{Fe}(\mathrm{CN})_{6}\right], 100 \mathrm{ml}$ distilled $\mathrm{H}_{2} \mathrm{O}$ (Fig. 2d and e) and third, $1 \mathrm{~g}\left(\mathrm{NH}_{4}\right) \mathrm{MoO}_{4}, 6 \mathrm{~g} \mathrm{NH} \mathrm{NH}_{4}, 200 \mathrm{ml}$ distilled $\mathrm{H}_{2} \mathrm{O}$ (Fig. 2b and c) [17]. All photomicrographs were taken using Differential Interference Contrast (DIC) microscopy.

The macrostructure of the LSP treated joint is presented in Fig. 2a; FSW typical regions, namely the heat affected zone, HAZ, the thermomechanically affected zone, TMAZ, and the weld nugget, WZ, are visible. The variation in grain size of each region of the joint depends on the base material and FSW processing parameters [11-12,18]. This large variation in textures across the weld zone is suggesting a consequent strong variation in local mechanical properties.

A larger TMAZ is found in the retreating side of the joint, as a peculiarity of the FSW process. The weld nugget appears wider on the crown region of the weld since the upper surface was in contact with the tool shoulder (Fig. 2a). At macro-scale, the diffuse onion rings are visible inside the weld zone. The nugget region
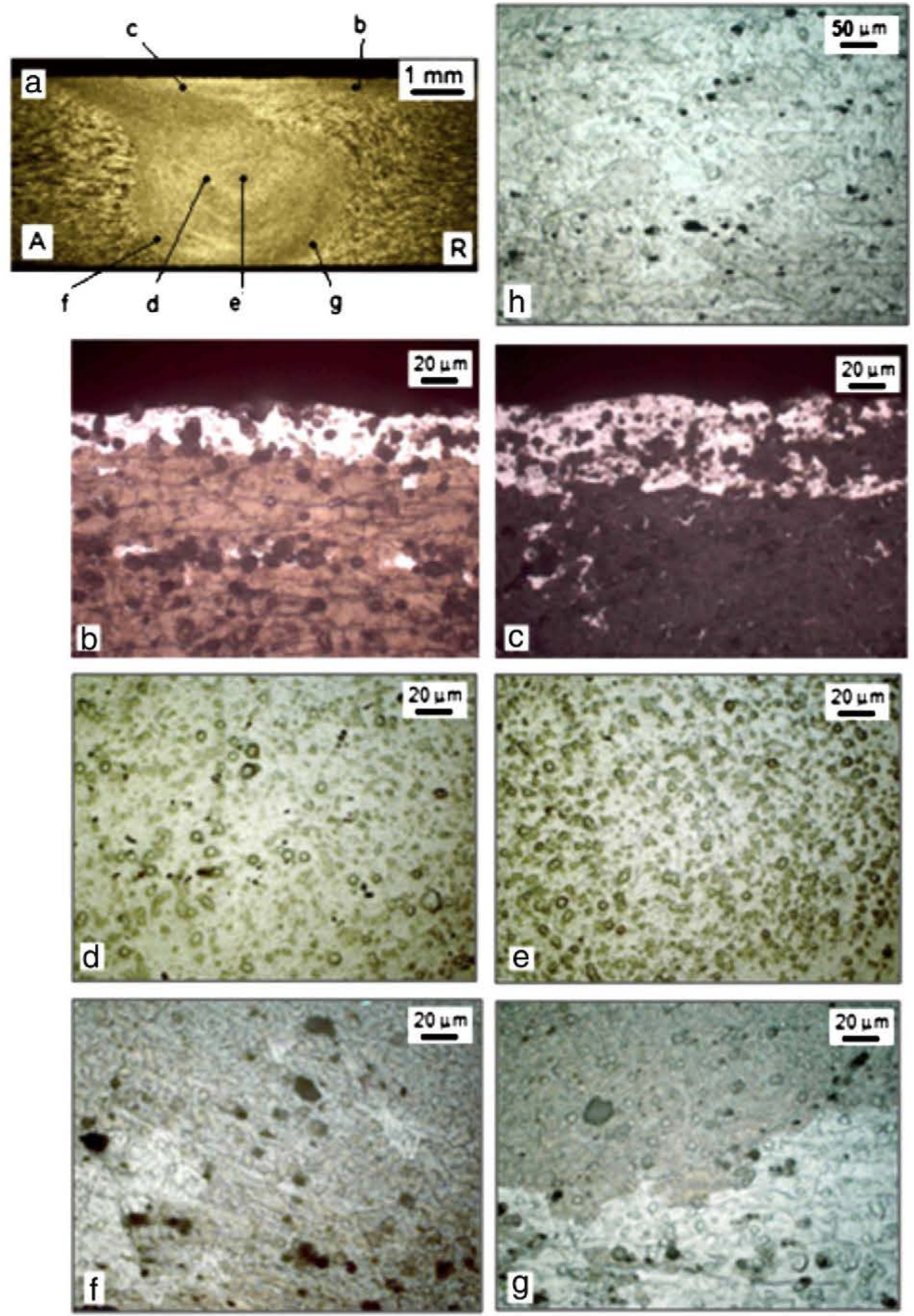

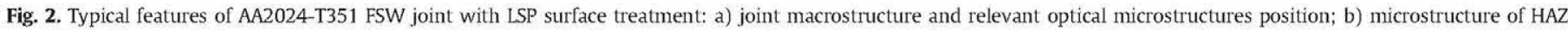

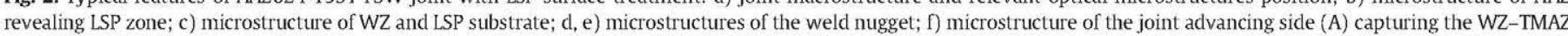
transition; $g$ ) microstructure of the joint retreating side (R) capturing the WZ-TMAZ transition; $h$ ) base material microstructure. 
a

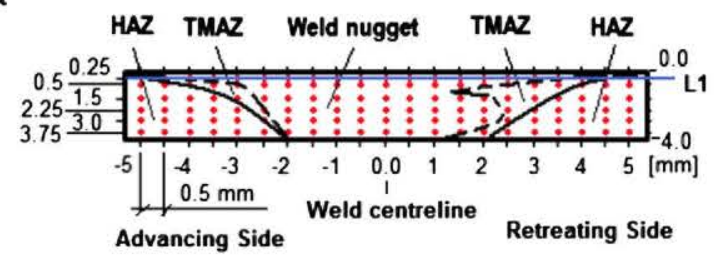

b

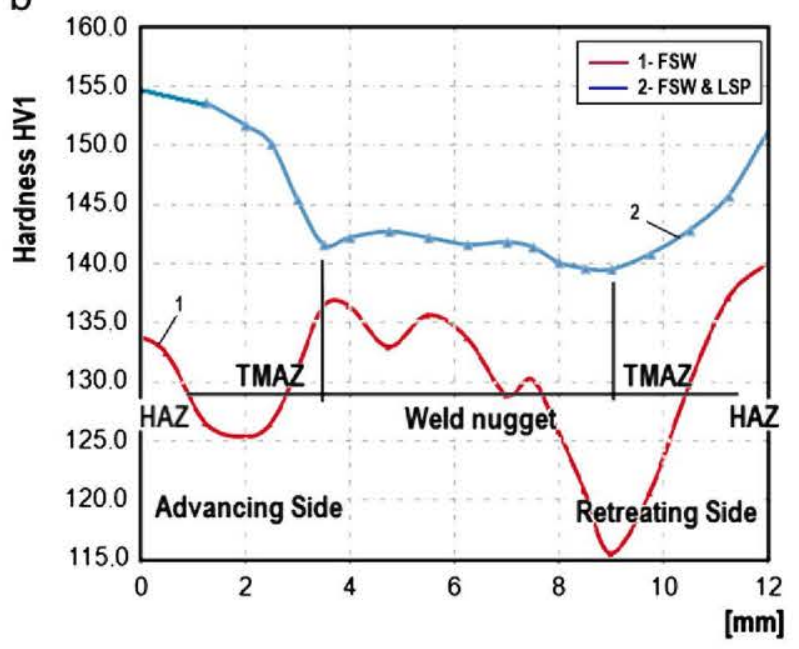

Fig. 3. Microhardness transverse across the FSW joint at $0.25 \mathrm{~mm}$ depth from the weld top surface and LSP influence: a) map of hardness measurements location; $L_{1}-$ line of considered hardness imprints; b) hardness profiles along $\mathrm{L}_{1}$ in case of: 1 - FSW joint; 2 - FSW joint previously treated by LSP on both sides.

microstructure (Fig. $2 \mathrm{~d}$ and e) indicates that the weld centre was subjected to high plastic deformation at high enough temperature to develop a fine grain structure by dynamic recrystallisation. The average grain size of this area of $6 \mu \mathrm{m}$ is in agreement with previously reported data $[11-12,19]$. TMAZ shows an elongated grain structure that is due to the severe plastic deformation that takes place during welding; the stirring action causes the flat grains of the parent metal to be drawn into and around the nugget zone (Fig. 2a, f, and g). The presence of the coarsened precipitates of $\mathrm{Al}_{2} \mathrm{CuMg}$ in $\mathrm{TMAZ}$ indicates that this zone is somewhat softer than the weld zone. The grain size in TMAZ is approximately 200 to $400 \mu \mathrm{m}$, slightly bigger than that of the base material. The HAZ microstructure (Fig. 2b) is unaffected by the mechanical effects from the welding tool and its structure resembles the pan-cake parent material, although the precipitates in this region are generally larger than in the base material; the grains size is about $150 \mu \mathrm{m}$. As reference, Fig. $2 \mathrm{~h}$ shows the base material (AA2024-T351) microstructure.

The LSP effects are presented in Fig. $2 \mathrm{~b}$ and $\mathrm{c}$, which show the upper side of the joint. Because flowing water was used as confining medium during LSP, the aqueous solution of ammonia molybdate [17] was found appropriate to reveal the LSP influence through the joint thickness; a highly oxidized, quasi-homogeneous layer was found, of approximately $20 \mu \mathrm{m}$ thick in HAZ, and of $40 \mu \mathrm{m}$ thick in WZ; this dimensional mismatch is due to the differences in grains size and orientation at the joint surface. Nevertheless, the oxidation is caused by the superficial interaction of the water-confined plasma with the substrate and has beneficial effects due to well-known properties of alumina.

\subsection{Hardness analysis}

Fig. 3a is presenting the map of Vickers hardness measurements made across the joint. The results are in agreement with previous investigations [12,19]. Data corresponding to L1 line, located at $0.25 \mathrm{~mm}$ depth from the weld top surface was considered relevant for understanding the influence of LSP close to the sample surface.

The values of the microhardness field across the native FSW joint are presented in Fig. 3b. The weld nugget, TMAZ and the HAZ microhardness values are gradually softer if comparing to the $140 \mathrm{HV} 1$
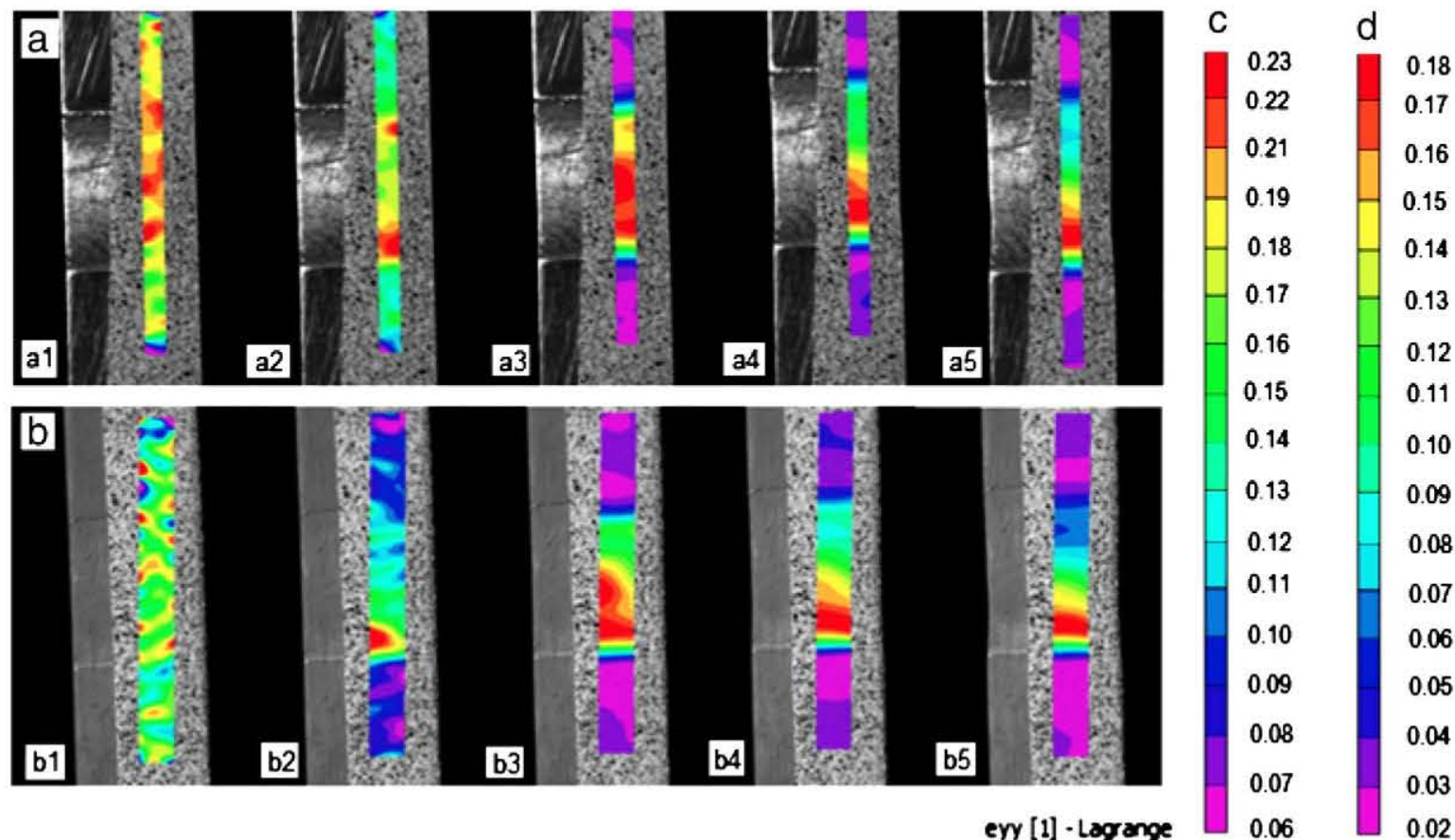

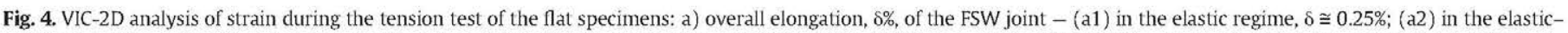

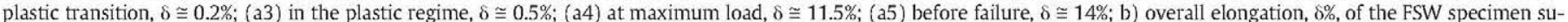

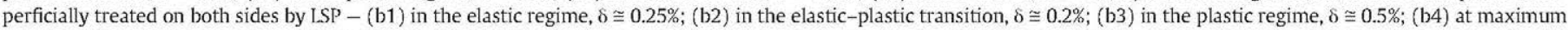

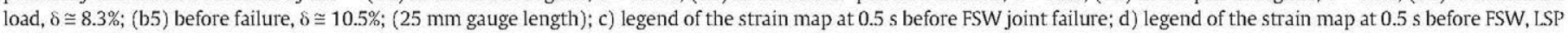
treated joint failure. 
average value of the native AA2024 alloy, due to the competing effects of dissolution and re-precipitation. In the weld nugget, the initial microstructure of the base material has been replaced by fine equiaxed grains. The data are consistent with previously observed $[4,6]$ under-matching between the weld and the base metal. The weld region exhibits the "W"-shaped hardness distribution which is typical for many friction stir welded joints when performed in precipitation hardening alloys. The lower temperatures of HAZ makes the initial precipitation hardening treatment of the base material to be more effective, explaining its higher hardness values when comparing with that of TMAZ. The asymmetry in size and hardness of the retreating side (RS) of TMAZ, when comparing with the advancing side (AS) of TMAZ, suggests that this region of material undergoes different thermo-mechanical cycles with respect to the tool pin rotation and weld direction $[11,20]$. The macrostructure from Fig. $2 a$ and the exhaustive examination of the sample with the optical microscope revealed relatively small and diffuse precipitates in the RS of TMAZ, comparing with the sharp and larger precipitates, transversely oriented to the joint in the AS of TMAZ, explains the lower hardness levels found in the RS, when comparing with the AS data. Its softening is due to the dissolution and coarsening of strengthening precipitates during the thermal cycle, by over-ageing. The minimum hardness values of this region indicate maximum strain concentration $[11,19]$, leading to a local degradation of the joint mechanical properties.

The transverse microhardness field (at $0.25 \mathrm{~mm}$ depth from the top side of the specimens), after LSP treatment (applied on both faces of the joint) is also presented in Fig. 3. The minimum hardness values were found in the TMAZ corresponding to the retreating side of the FSW joint. LSP produces the hardness increase in the weld area up to values comparable with those of the base metal (140 HV1). Since LSP was applied starting from the base metal unaffected by FSW, a superficial hardening (155 HV1) was noticed in this zone. The superficial hardening induced by the LSP treatment was found up to $1 \mathrm{~mm}$ depth from the specimens' top side, but its effects are clearly relevant on $0.25-0.3 \mathrm{~mm}$ depth [3-4,11,13-14].

\subsection{Tension test - local strain and overall results}

Fig. 4 presents the strain distribution measured with the VIC-2D technique during the tensile loading of the FSW flat specimens, native and superficially treated by LSP. Both tests were performed at a constant crosshead speed of $1 \mathrm{~mm} / \mathrm{min}$. The analysis was made on digital images continuously acquired at every $0.5 \mathrm{~s}$. The failure of the specimens was recorded at overall elongations of $14 \%$ in case of the FSW joint, and $10.5 \%$ for the LSP treated ones (Fig. 4). This difference of approximate $-3.5 \%$ reflects the LSP effect on the joint overall elongation. The images a1-a5 (Fig. 4a), indicate the location of maximum strain along the tensile test of the FSW sample: in the WZ and advancing side of TMAZ (a1), in both sides of TMAZ (a2), in the WZ and retreating side of TMAZ (a3), in the retreating side of TMAZ (a4), and (a5). The highest strain during the test was $\boldsymbol{\varepsilon}_{\max }=0.23$; it occurred just before tensile failure, in the retreating side of the TMAZ, indicating that this zone of the joint is the weakest (Fig. 4c). These results are consistent with the microhardness measurements and the macro/microstructure observations which are indicating that the original structure of this region is over-aged. Therefore, this area is relatively ineffective in inhibiting dislocation motion and the consequent strain localization here results into the joint failure [18].

In case of the tensile test of the LSP treated joint the location of maximum strain is shown in Fig. 4, b1-b5: dispersed in different joint areas (b1), in the retreating side of TMAZ (b2), in the WZ and retreating side of TMAZ (b3), and (b4) and in the retreating side of TMAZ (b5). The highest strain value $\left(\varepsilon_{\max L S P}=0.18\right)$ during the test occurred in the retreating side of TMAZ just before tensile failure (Fig. 4d).
These results indicate that LSP induces a planar field of compressive residual stresses, on both sides of the specimen, ranging from a maximum value at surface to zero at about $0.8-1 \mathrm{~mm}$ depth [3-8]. The difference of $22 \%$ between the maximum strains at joints failure can be attributed to compressive strain hardening induced by LSP.

The last strain field registries explain the failure occurrence in the retreating side (Fig. $4 \mathrm{a} 5$ and b5) of both types of joints. These strain fields are also showing that the tensile behavior of the welded joints is not symmetric in the two interfaces of the weld ( $A$ and $R$ ), and that the retreating side (RS) is weaker than the advancing side (AS).

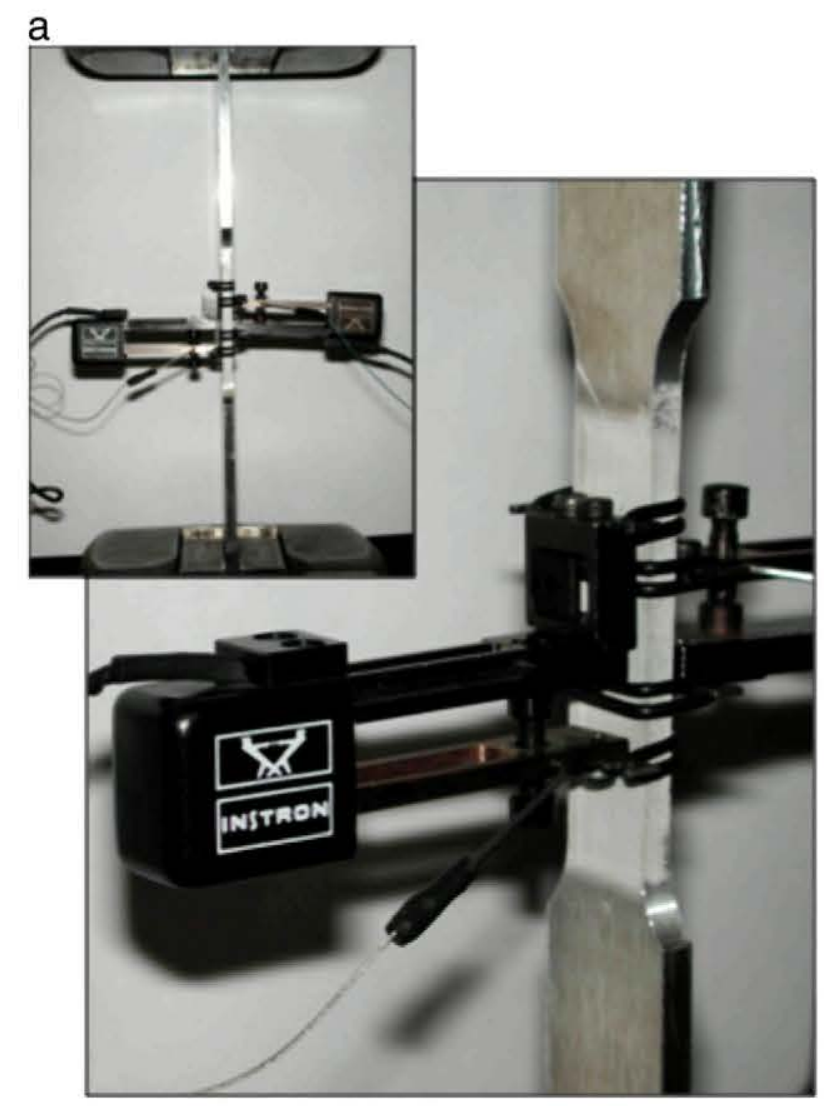

b

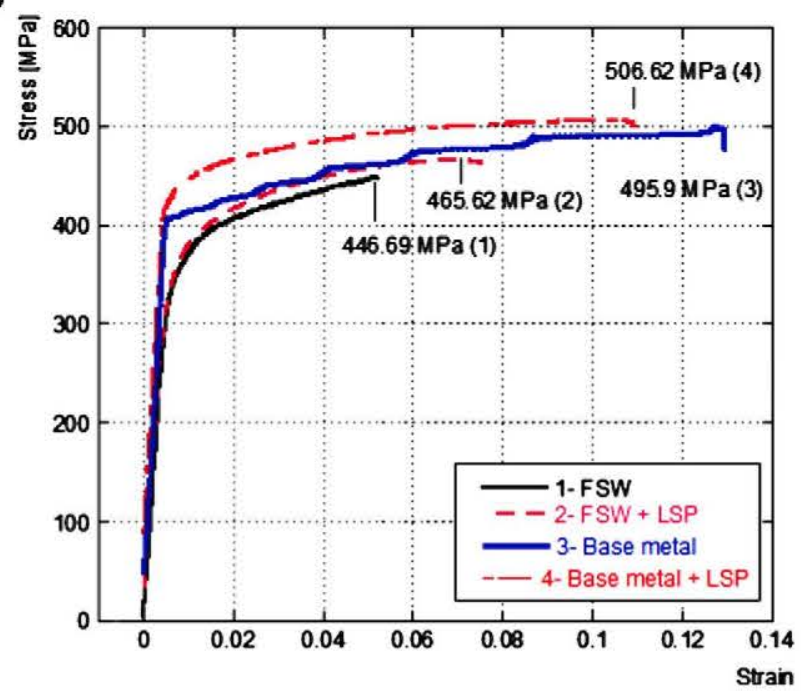

Fig. 5. Tension tests - overall results: a) extensometers position; b) engineering stress-strain curves of: 1 - FSW joint; 2 - FSW joint with LSP surface treatment; 3 - native base metal AA2024-T351; 4 - AA2024-T351 with LSP surface treatment 
Engineering stresses for a given level of strain are lower in the welded joint than in the base material (BM).

Engineering stress-strain curves of FSW joints and base metal, both LSP treated and untreated, were obtained from tensile tests carried out with direct elongation measurements by conventional clipon extensometers. These curves are presented in Fig. 5. The failure of both types of welded specimens was located in/at TMAZ interface with the weld nugget (WZ), on the retreating side at a $45^{\circ}$ angle with respect to the plate surface. It results from the plastic strain concentration on the RS of TMAZ due to the strength mismatch between the base metal and the joint regions.

The welded joint is inhomogeneous (Fig. 2), and the conventional tension test provides information only on the overall stress-strain behavior, from which it was found that the overall loss in tensile strength of the FSW sample is $10 \%$ with respect to the BM. Samples superficially treated with LSP shown better tensile behavior, and only a 5\% decrease in tensile strength vs. BM was encountered. As shown in Fig. 2, LSP treatment increases the overall ductility of treated FSW joints.

The changes in the overall mechanical behavior of the welded joint due to LSP are induced by superficial hardening of the joint top and bottom faces.

VIC-2D dedicated software was used to simulate 4 virtual extensometers on different zones of the FSW joint (Fig. 6a1). The strains were obtained by averaging the displacement field on the gauge length, while the stresses were conventionally computed from the measured tension loads. Thus, local strain-stress curves of different joint zones and base metal were obtained from a single tension test (Fig. 6b). These curves are presenting information on tensile behavior
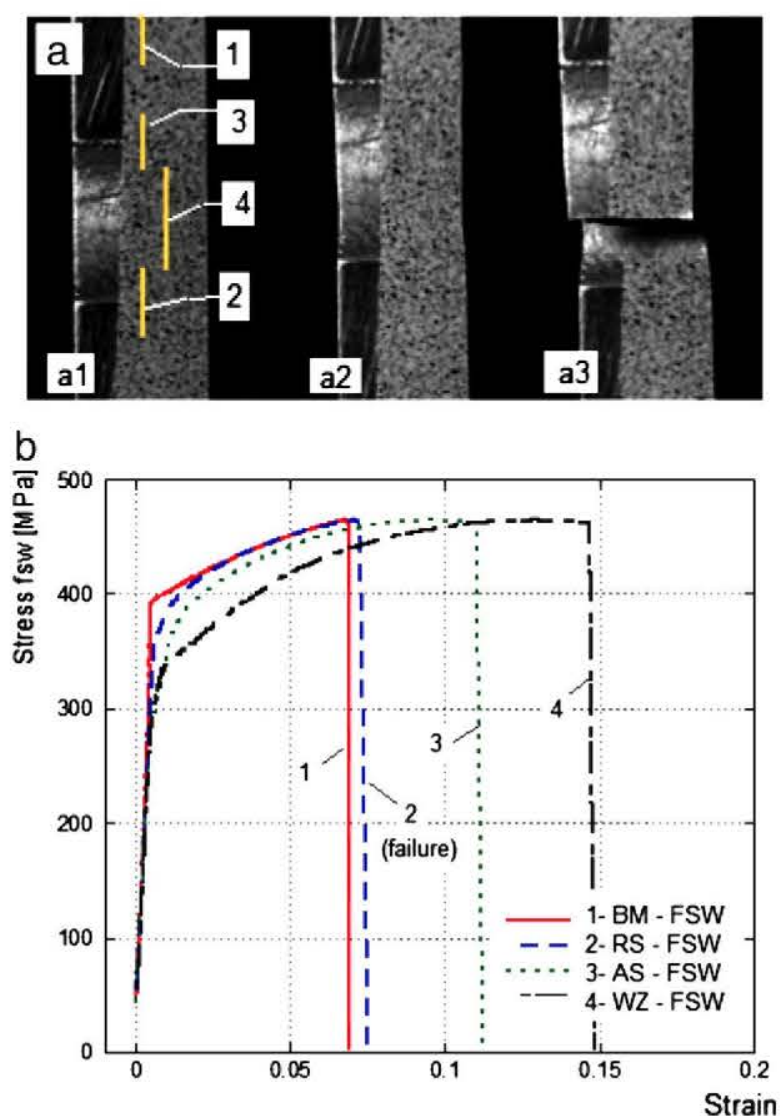

Fig. 6. VIC-2D overall tension test results on FSW native joint: a) speckle image showing: the initial gauge (virtual extensometer) positions and lengths (a1), joint maximum elongation (a2), failure position (a3); b) engineering stress-strain curves of: 1 - native base metal AA2024-T351; 2 - retreating side of the FSW joint; 3 advancing side of the joint; 4 - weld nugget. of the BM, WZ an AS regions up to the specimen failure in the RS of the FSW joint. According to these curves, the material of BM, WZ and AS is experiencing some plastic deformation, but, it does not reach the local tensile strength; VIC cannot provide information beyond the specimens' failure in the RS. Fig. $6 \mathrm{a} 2$ and a3 shows the specimen at maximum deformation and failure, respectively. The joint fails at the retreating side of TMAZ well before its tensile instability, when the native BM is quite far from it, and the WZ and the advancing side of TMAZ have widely surpassed it.

The same type of analysis was made for the FSW joints, LSP treated. Fig. 7a shows the initial virtual gauge lengths and the specimen images at maximum deformation and failure newly occurred at the interface of the weld nugget and the retreating side of TMAZ. Fig. 7b illustrates the strain-stress curves from different regions of the FSW, LSP treated joint, providing information on tensile behavior of the $\mathrm{BM}, \mathrm{WZ}$ an $\mathrm{AS}$ regions up to the specimen failure in the RS. It was found that LSP delays the joint failure beyond tensile instability of the retreating side of TMAZ, by increasing with 30\% the failure strain of this zone (Fig. 8a).

Fig. $8 \mathrm{~b}$ shows the mechanical properties of different regions of the FSW joints, native or LSP treated obtained using VIC "virtual extensometers" by assuming that the transversely loaded specimens are in iso-stress configuration. It was found that the yield strength (YS) of the FSW joint decreases when comparing with the native BM, with $16 \%$ in the AS, with $20 \%$ in the WZ and with $21 \%$ in the RS. This is due to the large variations in residual stress, hardness and microstructure as the weld line is traversed [18-20]. The compressive stresses added by LSP are minimizing the differences in YS between the
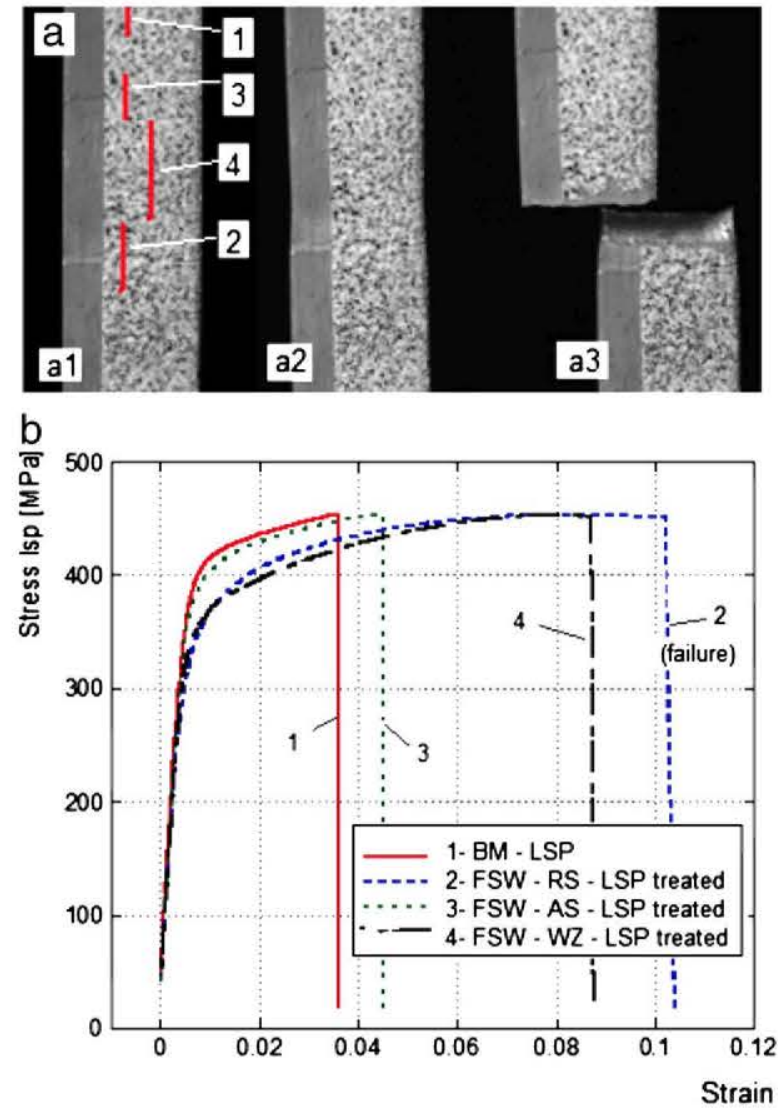

Fig. 7. VIC-2D overall tension test results of the FSW joint with LSP surface treatment: a) speckle image showing: the initial gauge (virtual extensometer) positions and lengths (a1), joint maximum elongation (a2), failure position (a3); b) engineering stress-strain curves of: 1 - native base metal AA2024-T351 with ISP; 2 - retreating side of the FSW joint with LSP; 3 - advancing side of the joint with LSP; 4 - weld nugget with LSP. 
native $\mathrm{BM}$ and the weld regions; the decrease in YS was of $10 \%$ in the AS, $17 \%$ in the WZ and $18 \%$ in the RS. LSP is increasing with $6 \%$ the YS of the RS when comparing with the as welded data. It results that the main benefit of LSP is the increase, of different magnitudes, of the YS of the weld different regions.

The joints, native FSW and LSP treated have to be understood as composite materials, resulting from the combination of 4 distinct regions, where the overall strain/elongation is the average of the correspondent elongations at joint failure in tension. Their tensile failure is governed by the combination of these regions of different strength, the weakest determining the fracture load. LSP superficial treatment applied on the whole FSW joint area produces a significant increase of the tensile loading capacity, and also a presumable increase of fatigue and corrosion resistance $[18,20]$. The compressive residual stress field resulting from LSP contributes to the structure servicelife by delaying the growth of eventual surface flaw generated in the presence of specific environments [20-21].

\subsection{Stress corrosion cracking (SCC) susceptibility}

Slow strain rate tensile tests (SSRT) with the joints immersed in a $3.5 \% \mathrm{NaCl}$ solution at room temperature were carried out at a strain rate of $1 \times 10^{-6} \mathrm{~s}^{-1}$. The specimen geometry was that of Fig. 1. A sealed cell containing the corrosive solution and the welded zone of
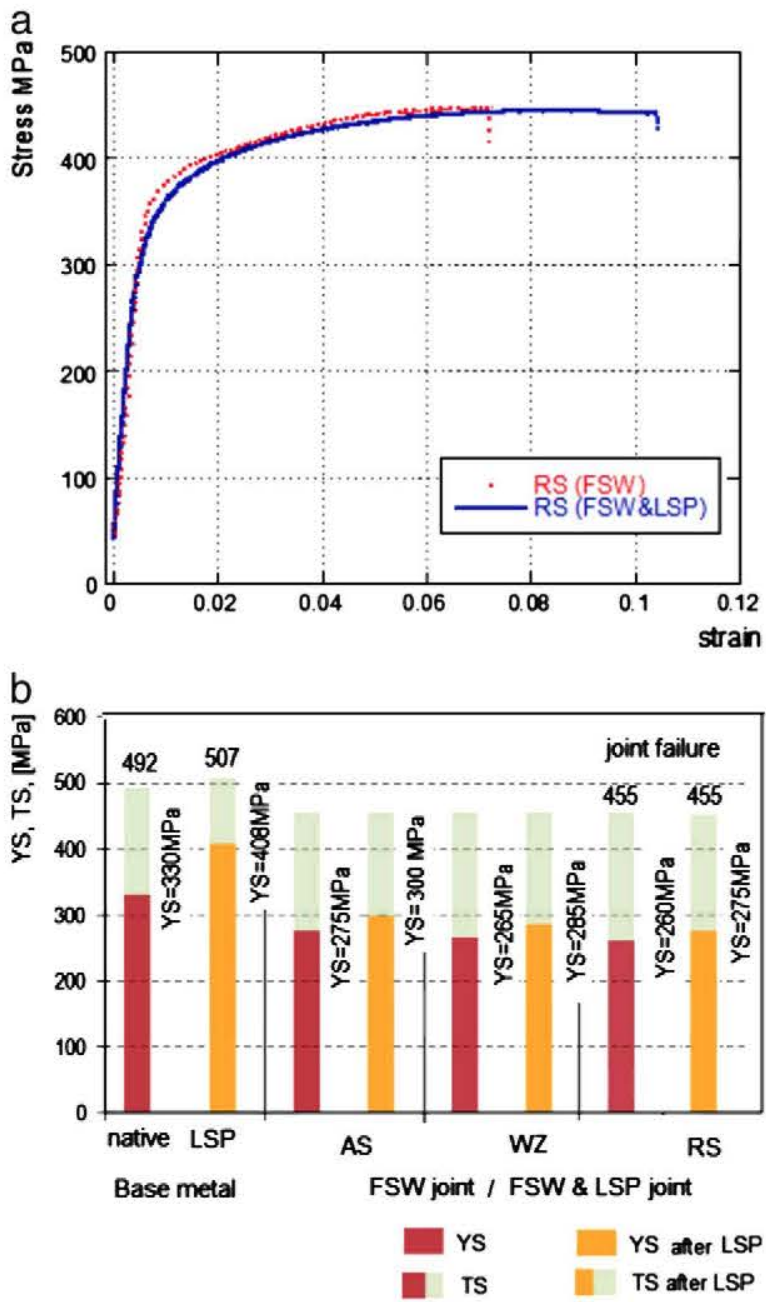

Fig. 8. LSP influence on the FSW joint properties: a) stress-strain curves in the RS of the joint (1 - as welded vs. 2 - LSP treated); b) yield strength (YS) and tensile strength (TS) properties of the BM and FSW regions native or LSP treated (advancing side AS, weld nugget $-\mathrm{WZ}$ and retreating side - RS).
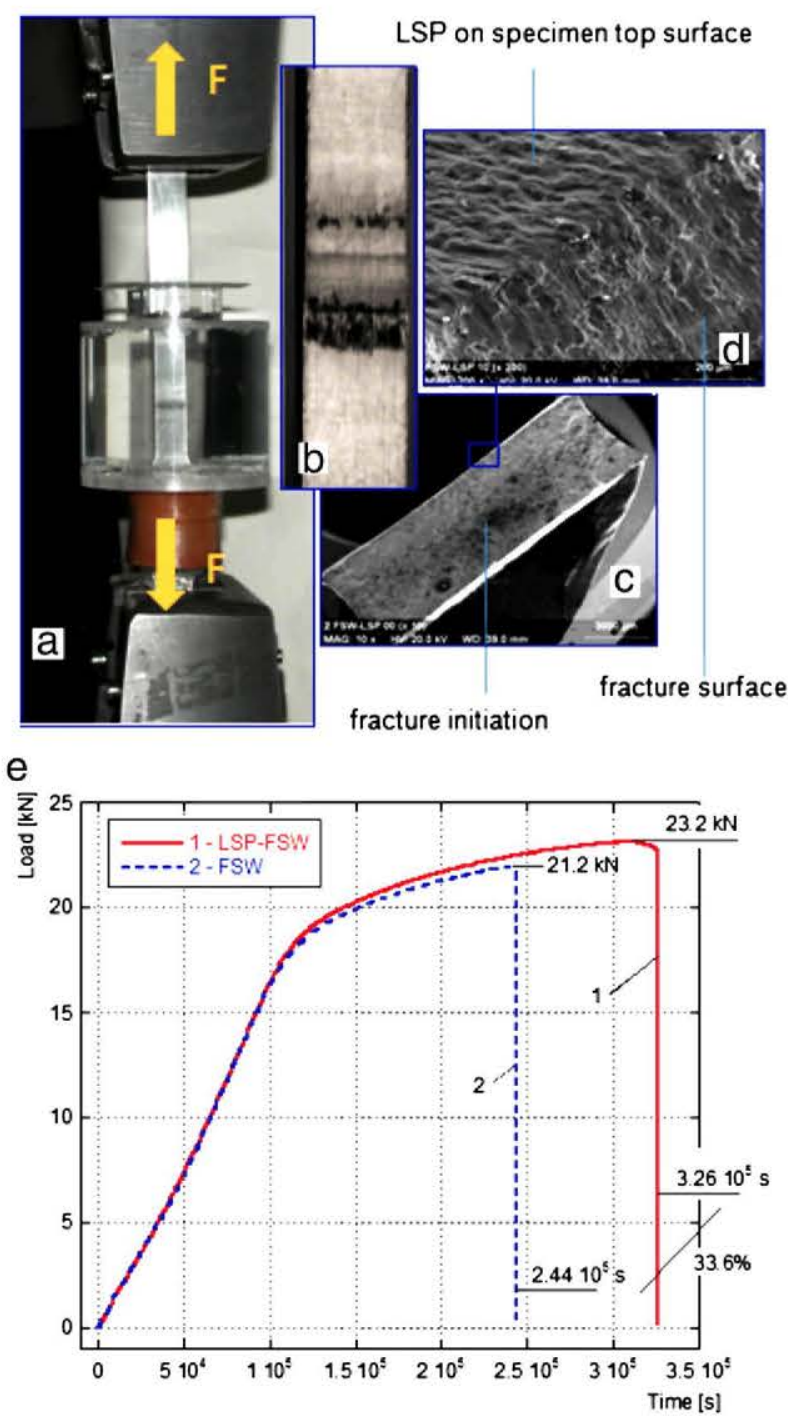

Fig. 9. LSP influence on FSW joints - SSRT in a $3.5 \% \mathrm{NaCl}$ solution: a) Image captured during the SSRT of the un-treated FSW joint; b) FSW joint, ISP treated during SSRT; c) SEM image showing the fracture initiation of the FSW specimen, LSP treated; d) SEM image showing the end of the fracture and the LSP top side of the sample; e) SSRT results (Load vs. time curves): 1 - FSW joint with LSP surface treatment; $2-$ FSW joint.

the specimens was placed in the testing machine, as shown inFig. 9a. The test results are presented in Fig. 9e.

All tested specimens fractured at the interface between the weld nugget and the TMAZ in the retreating side of the FSW joint; no sign of stress corrosion cracking was found on the specimen surfaces in contact with the aggressive solution (Fig. 9c and d).

However, signs of active pitting corrosion initiation were visible on the TMAZ of the FSW un-treated specimen after $48 \mathrm{~h}$ of testing (Fig. 9b). In spite of this pitting attack initiation, the results indicate that the general corrosion rates were very small for all tested samples. No signs of pitting corrosion were found on LSP treated samples. This is due to the compressive residual stress induced by LSP on the specimen surface and through its thickness, as too [21].

The only notable difference between the LSP treated and untreated FSW joints was the recorded load vs. time curves. Failure loads slightly differ, but failure time is $33 \%$ higher for the LSP treated joint. This indicates that the joint surface layers due to LSP treatment are delaying the damage mechanisms involved in SCC. 


\section{Conclusions}

VIC method was proved efficient for assessing the mechanical behavior of local zones of an inhomogeneous material, such as the FSW joint. VIC is a feasible method to experimentally analyze tensile behavior of local areas of reduced dimension structural joints.

At joint scale, a surface hardening effect is induced by LSP affecting the overall mechanical behavior of the FSW joint.

LSP is positively influencing the FSW joint overall plastic deformation in the uniform elongation zone and delaying its failure beyond the ultimate tensile strength.

The overall ductility of the FSW joints is increased by the LSP treatment. The plastic strain concentration at the retreating side of TMAZ causes failure. This is due to the strength mismatch between the base metal and the joint regions.

The main benefit of LSP is the increase, of different magnitudes, of the YS of the weld different regions. LSP delays the joint failure beyond tensile instability in the RS of TMAZ, by increasing with $30 \%$ the strain at failure.

The low strain rate tensile testing of the FSW joints in an aggressive medium shows that LSP treatment inhibits the pitting corrosion initiation observed in the un-treated FSW joints. The compressive residual stresses induced by LSP are mitigating the tensile residual stress of FSW, delaying the action of the damage mechanisms involved in stress corrosion cracking.

Concluding, the overall effect of LSP is the increase of the tension loading capacity of the FSW joints as well as a presumable important increase of fatigue and stress corrosion resistance.

\section{Acknowledgements}

The authors gratefully acknowledge the partial financial support of the Spanish Ministerio de Ciencia e Innovacion through the projects CONSOLIDER-INGENIO 2010 CSD00C-06-14102, BIA200806705-C02-01, and SHOCKLAS+/MAT2008-02704/MAT. The authors acknowledge the essential contribution of Dr. Pedro Vilaça and Eng. Catarina Vidal, who were with DEM-Instituto Superior Tecnico, TULISBON, Lisbon and kindly provided the FSW welded joints.

\section{References}

[1] P.S. De, R.S. Mishra, Sci. Technol. Weld. Joining 16 (4) (2011) 343.

[2] R.S. Mishra, Z.Y. Ma, Mater. Sci. Eng., R 50 (1-2) (2005) 1.

[3] K. Surekha, B.S. Murty, K. Prasad Rao, Sutf. Coat. Technol. 202 (2008) 4057

[4] O. Hatamleh, P.M. Singh, H. Garmestani, J. Mater. Eng. Perform. 18 (4) (2009) 406.

[5] M. Iordachescu, D. Iordachescu, M. Blasco, E. Scutelnicu, Metal. Int. 14 (7) (2009) 99.

[6] M.B. Uday, M.N. Ahmad Fanzi, H. Zuhailawati, A.B. Ismail, Sci. Technol. Weld. Joining $15(7)(2010) 534$

[7] K. Colligan, Weld. J. 75 (7) (1999) 229s.

[8] O. Hatamleh, S. Forth, A.P. Reynolds, J. Mater. Eng. Perform. 19 (1) (2010) 99

[9] P. Staron, M. Kocak, S. Williams, A. Wescott, Phys. B Condens. Matter 350 (1-3) (2004) e491.

[10] L. Fratini, B. Zuccarello, Int. J. Mach. Tools Manuf. 46 (6) (2006) 611.

[11] M.A. Sutton, A.P. Reynolds, D.-Q. Wang, C.R. Hubbard, J. Eng. Mater. Technol. 124 (2002) 215.

[12] A. Ali, M.W. Brown, C.A. Rodopoulos, S. Gardiner, J. Fail. Anal. Prev. 6 (4) (2006) 83.

[13] J.L. Ocaña, M. Morales, J.A. Porro, C. Molpeceres, D. Iordachescu, Metal. Int. 14 (3) (2009) 113.

[14] M. Morales, J.L. Ocaña, C. Molpereces, J.A. Porro, A. Garcia-Beltrán, Surf. Coat. Technol. 202 (2008) 2257

[15] S. Bagherifard, M. Gualiano, Surf. Eng. 25 (11) (2009) 1.

[16] Designation and Chemical Composition Limits for Wrought Aluminium and Aluminium Alloys. The International Alloy Designation System (IADS), Aluminium Association of the United States, USA, 1998.

[17] ASM Handbook, Metallography \& Microstructures, Vol. 9, 2004, p. 525

[18] O. Hatamleh, A. DeWald, J. Mater. Process. Technol. 209 (2009) 4822

[19] G. Bussu, P.E. Irving, Int. J. Fatigue 25 (2003) 77.

[20] O. Hatamleh, J. Lyons, R. Forman, Int. J. Fatigue 29 (2007) 421.

[21] O. Hatamleh, P.M. Singh, H. Garmestani, Corros. Sci. 51 (2009) 135. 